\title{
Analysis of the pathogenic variants of $B R C A 1$ and BRCA2 using next-generation sequencing in women with familial breast cancer: a case-control study
}

Omar Alejandro Zayas-Villanueva², Luis Daniel Campos-Acevedo', José de Jesús Lugo-Trampe", David Hernández-Barajas², Juan Francisco González-Guerrero², María Fernanda Noriega-Iriondo², Ilse Alejandra Ramírez-Sánchez ${ }^{1}$ and Laura Elia Martínez-de-Villarreal ${ }^{1 *}$ (D)

\begin{abstract}
Background: Pathogenic variants (PVs) of BRCA genes entail a lifetime risk of developing breast cancer in 50-85\% of carriers. Their prevalence in different populations has been previously reported. However, there is scarce information regarding the most common PVs of these genes in Latin-Americans. This study identified BRCA1 and BRCA2 PV frequency in a high-risk female population from Northeastern Mexico and determined the association of these mutations with the patients' clinical and pathological characteristics.

Methods: Women were divided into three groups: aged $\leq 40$ years at diagnosis and/or risk factors for hereditary breast cancer $(n=101)$, aged $>50$ years with sporadic breast cancer $(n=22)$, and healthy women $(n=72)$. Their DNA was obtained from peripheral blood samples and the variants were examined by next-generation sequencing with lon AmpliSeq BRCA1 and BRCA2 Panel using next-generation sequencing.
\end{abstract}

Results: PVs were detected in 13.8\% group 1 patients (BRCA1, 12 patients; BRCA2, 2 patients). Only two patients in group 2 and none in group 3 exhibited BRCA1 PVs. Variants of uncertain significance were reported in 15.8\% patients $(n=16)$. In group 1, patients with the triple-negative subtype, PV frequency was $40 \%(12 / 30)$. Breast cancer prevalence in young women examined in this study was higher than that reported by the National Cancer Institute Surveillance, Epidemiology (15.5\% vs. 5.5\%, respectively).

Conclusions: The detected BRCA1 and BRCA2 PV frequency was similar to that reported in other populations. Our results indicate that clinical data should be evaluated before genetic testing and highly recommend genetic testing in patients with the triple-negative subtype and other clinical aspects.

Keywords: Pathogenic variant, BRCA1 and BRCA2 genes, Triple-negative subtype, Hereditary, Breast cancer

\section{Background}

Breast cancer is the most common type of cancer among women worldwide and is the main cause of death in developing countries. In 2012, 1.67 million cases were reported worldwide by GLOBOCAN. Hereditary and familial cancers represent approximately $10 \%$ of the cases,

\footnotetext{
* Correspondence: laelmar@yahoo.com.mx

'Department of Genetics, Hospital Universitario "José E. González", Monterrey, Mexico

Full list of author information is available at the end of the article
}

indicating that 167,000 cases may be attributed to a genetic cause [1].

Approximately $15-40 \%$ of hereditary breast cancers occur due to pathogenic variants (PVs) of BRCA1 (17q21) and BRCA2 (13q12-13) [2-5]. BRCA PVs may be present in one of eight breast cancer patients aged $<40$ years and who have two affected relatives [3]. Carriers of PVs of BRCA genes have a $60 \%$ risk of developing breast cancer at the age of 70 years and an $83 \%$ risk of developing contralateral breast cancer [6]. Ovarian cancer has high penetrance and association with BRCA PVs. Several other

(c) The Author(s). 2019 Open Access This article is distributed under the terms of the Creative Commons Attribution 4.0 International License (http://creativecommons.org/licenses/by/4.0/), which permits unrestricted use, distribution, and reproduction in any medium, provided you give appropriate credit to the original author(s) and the source, provide a link to the Creative Commons license, and indicate if changes were made. The Creative Commons Public Domain Dedication waiver (http://creativecommons.org/publicdomain/zero/1.0/) applies to the data made available in this article, unless otherwise stated. 
malignancies, such as pancreatic cancer, prostate cancer, and melanoma, have also been associated with mutations in BRCA genes; hence, the patients' family history should be considered.

The prevalence of $B R C A 1 / 2$ germline mutations varies among ethnic groups and geographical zones. Clear variability across Latin American countries has been described, which is explained by the mixture of European, African, and Amerindian ancestors [7]. A founder mutation, ex9-12del, has been described in the Hispanic population from the south of the United States [8], and in an unselected study population from the center of Mexico that was assessed for a family history of cancer and exhibited a mutation frequency of $29 \%$ [9]. Mexico is a genetically heterogeneous country, and BRCA PVrelated information obtained using next-generation sequencing (NGS) is scarce. PVs should be identified for better disease characterization among different populations and for appropriate genetic counseling.

This study established the frequency and type of mutations of BRCA1 and BRCA2 in a female population from Northeastern Mexico and determined the correlation of mutations with the patients' clinical and pathologic characteristics.

\section{Methods}

We performed a case-control study comprising patients from the Centro Universitario Contra el Cáncer at the Hospital Universitario Dr. Jose E. Gonzalez from the Universidad Autónoma de Nuevo León. Subjects (including their parents and grandparents) from Northeastern Mexico (Nuevo León, Tamaulipas, and Coahuila) with high-risk factors for hereditary breast cancer. Enrollment strategy included searching on local data base from January 2005 to August 2015. Women with breast cancer at early age $(\leq 40 \mathrm{y})$ were invited to participate in our study.

Sample size for case-control design considering alpha error 0.05 and beta of 0.8 resulted in 25 persons per group. Despite the calculated sample size, a pre-planned enrollment to recruit 200 people was conducted.

In addition to having a pathological diagnosis of breast cancer, patients were required to meet at least one of the following criteria: age $\leq 40$ years at diagnosis [10]; presence of bilateral breast cancer; and three or more relatives with breast cancer, ovarian cancer, pancreatic, prostate, or melanoma cancer; the latter two were independent criteria that did not consider age at diagnosis to be $<40$ years.

We include two control groups. Patients with a diagnosis of sporadic breast cancer were termed "positive controls," and healthy women without a personal or family history of cancer were termed "negative controls." For the last group, an open invitation was made to medical students and workers for detecting local variants. Inclusion criteria for the healthy group included the following: $>18 \mathrm{y}$, pedigree with no personal or family history of any cancer, born in the Northeast of Mexico. Informed consent was required for all included patients. Patients meeting the inclusion criteria were selected from the daily hospital outpatient attendance register or from the electronic database of the center and invited to participate by phone. Healthy controls were selected from the general population. An oncologist conducted an interview to obtain the medical history. Clinical data were verified from the electronic medical files of the patients and recorded as baseline data. Peripheral blood sample was taken and analyzed at the molecular laboratory of the genetics department in the university hospital (College of American Pathologists accredited).

\section{Pathology and mutation analyses}

All patients (cases and positive controls) received a diagnosis of invasive breast cancer that was confirmed by anatomopathological analysis at the pathology department of the university hospital. The histologic type of the cancer was determined according to the World Health Organization system [11]. Tumor grade was defined using the Scarff-Bloom-Richardson system. Estrogen and progesterone receptors and HER2 were identified using standard immunohistochemical techniques; hormone receptors were considered positive when at least $1 \%$ stain was detected [12]; HER2 was considered positive when "+++" was detected; if "++" was observed, fluorescence in situ hybridization analysis was used for confirmation [13].

DNA extraction was performed using the Qiagen QIAamp DNA Mini Kit, (QIAGEN GmbH, Hilden, Germany), according to the manufacturer's instructions. Elution was into $100 \mu \mathrm{L}$ of water.

The entire coding regions of the BRCA1 and BRCA2 genes were amplified using the Ion AmpliSeq $B R C A 1$ and BRCA2 Panel (Life Technologies, Carlsbad, CA, USA) consisting of three primer pools, covering the target regions in 167 amplicons, including all exons and 10-20 bp of intronic flanking sequences, for both genes. Emulsion polymerase chain reaction (PCR) was performed using the Ion OneTouch ${ }^{\mathrm{m}} 2$ Instrument (Cat. No. 4474778), as indicated in Ion $\mathrm{PGM}^{\mathrm{m}}$ Template OT2 200 Kit (Publication Number MAN0007221. Rev.A.0; Cat. No. 4480974). Normalized 16-pM sample libraries were pooled and combined with OT2 kit reagents and Ion Sphere particles (ISPs) using an Ion OneTouch ES system (Life Technologies, Carlsbad, CA). Quality control was performed using the Ion Sphere ${ }^{\mathrm{Tm}}$ Quality Control kit (Life Technologies) to ensure that $10-30 \%$ of template-positive ISPs were generated in the emulsion 
PCR. After the ISP preparation, massively parallel paired-end sequencing was performed with an Ion Torrent Personal Genome Machine (PGM) system using the Ion PGM 200 Sequencing Kit and Ion 316 Chip (Life Technologies), according to the manufacturer's instructions.

For cases with negative findings, multiple ligationdependent probe amplification (MLPA) was performed to search for large genomic alterations, duplications, or deletions of one or more exons, as per guideline recommendations [14]. The SALSA MLPA P087-C1 BRCA1 and SALSA MLPA P077-A3 BRCA2 test kits (MRC-Holland, Amsterdam, Netherlands) were used in accordance with the manufacturer's instructions.

\section{Data analysis}

The raw data were analyzed using torrent suite software v5.0.4 (Life technologies). Coverage analysis was performed using the coverage analysis plug-in v5.0.2.0. Mutations were detected using the Variant Caller plugin v5.0.2.1 (Life Technologies). To eliminate erroneous base calling, two filtering steps were used to generate final variant calling. The first filter was set at an average total coverage depth of $>80$, each variant coverage of $>20$, a variant frequency of each sample of $>5$, and $p$-value of $<0.01$. The second filter was employed by visually examining mutations using Integrative Genomics Viewer software (https://software.broadinstitute.org/software/igv/). Ion Reporter 5.0 was used for variant annotation and classification.

After the filtrations, all variants identified through NGS (silent, missense, nonsense, frameshift, and splicing variants) were compared with variants in the 1000 Genomes Project (http://www.1000genomes.org/) for different ethnic populations, using ExAC (http://exac. broadinstitute.org/about) and 72 in-house controls. All mutations were also checked against the UMD, LOVD, kConFab, HGMD, and ClinVar databases, and were regarded as "pathogenic" if classified as such in these databases. The missense variants were annotated using the wANNOVAR web site (http://wannovar.wglab.org), which provides tools such as SIFT, PolyPhen-II HDIV, PolyPhen-II HVAR, LRT, Mutation Taster, Mutation Assessor, FATHMM, PROVEAN, VEST3, MetaLR and M-CAP to predict the effect of amino acid substitution for each missense mutation. Every missense mutation was scored as damaging or benign using the 11 prediction tools. If the missense mutation was scored as damaging by five or more of the prediction tools, the mutation was classified as a "damaging" mutation, and if it was scored by less than three, the mutation was classified as "benign". The detected variants are classified based on the criteria of the ENIGMA (Evidence-based Network for the Interpretation of Germline Mutant
Alleles) consortium (https://enigmaconsortium.org) and described as recommended by Human Genome Variation Society (https://www.hgvs.org/) using as RefSeq: NM_007294.3 and NM_000059.3. To verify if the PVs identified were true variants or sequencing artifacts, point mutations classified as PV were confirmed by Sanger sequencing, using the BigDye Terminator v3.1 sequencing kit and the ABI PRISM 3130 Genetic Analyzer (Life Technologies).

\section{Statistical analysis}

Patient characteristics were tabulated, and description data are presented as the mean with standard deviations and proportions. Comparisons between groups (familial hereditary vs. sporadic and carriers vs. noncarriers) were performed using a t-test for two independent means and chi-squared test for two proportions expressed as percentages. Odds ratios (ORs) were calculated for age, bilateral cancer, family history, and triple-negative variables. SPSS version 20 (IBM, Armonk, NY) for Windows 7 was used for statistical analysis.

\section{Results}

All subjects were born in Northeastern Mexico. From January 2005 to August 2015, 3,065 patients were registered in the hospital database. We eliminated 265 patients because the reported age was not reliable. There were 436 patients $(15.5 \%)$ aged $\leq 40$ years at diagnosis, among whom 335 were either not located or did not agree to participate. 101 patients were included with early age breast cancer and/or familial/hereditary breast cancer, 22 patients with sporadic cancer (positive controls), and 72 healthy women (negative controls). The clinical characteristics of the patients and positive control groups are shown in Table 1. As expected, the mean age of the familial breast cancer group was significantly lower ( $36.9 \pm 5.2$ years). No statistically significant differences were noted between the groups. Regarding tumor histopathology, $53 \%$ of patients in the hereditary cancer group exhibited nuclear grade 3 compared with only $10 \%$ in the sporadic cancer group $(p<0.001)$.

PGM sequencing of these 195 patients had an average of 60,463 reads per patients, with the mean read length being $113 \mathrm{bp}$. The average read depth per sample was $330 \mathrm{X}$, with the mean percentage of reads on target being $92 \%$ and uniformity of base coverage being $96.3 \%$. PV analysis of $B R C A 1$ and $B R C A 2$ revealed 16 mutation carriers. 14 carriers (13.8\%) present 10 different PVs in group 1 (Table 2). Overall, 12 different PVs were detected, and most of them (82\%) were of BRCA1 (13/16), whereas only $18 \%$ (3/17) were of BRCA2. Among these, 11 variants were classified as pathogenic and one as likely pathogenic. Sixteen variants were identified, eight (50\%) through NGS, and eight (50\%) using MLPA. PVs 
Table 1 Baseline characteristics of groups 1 \& 2; risk factors, tumor characteristics, and treatment

\begin{tabular}{|c|c|c|}
\hline \multirow[t]{2}{*}{ Characteristic } & Group 1 & Group 2 \\
\hline & $n=101$ & $n=22$ \\
\hline \multicolumn{3}{|l|}{ Age at diagnosis, } \\
\hline (years); $\pm S D$ & $36.9 \pm 5.2$ & $53.1 \pm 4.8$ \\
\hline \multicolumn{3}{|l|}{ Familial cancer } \\
\hline n (\%) & $54(53 \%)$ & 0 \\
\hline \multicolumn{3}{|l|}{ BMI, } \\
\hline mean; $\pm S D$ & $27.7 \pm 5.3$ & $27.1 \pm 4.2$ \\
\hline \multicolumn{3}{|l|}{ Age at menarche } \\
\hline mean $\pm S D$ & $12.3 \pm 1.4$ & $12.7 \pm 1.3$ \\
\hline \multicolumn{3}{|l|}{ Parity } \\
\hline mean $\pm S D$ & $2.4 \pm 1.6$ & $3.6 \pm 2.1$ \\
\hline Nuliparous; n (\%) & $18(18 \%)$ & $1(5 \%)$ \\
\hline \multicolumn{3}{|l|}{ Age at first pregnancy } \\
\hline mean $\pm S D$ & $21 \pm 5.4$ & $23 \pm 5.6$ \\
\hline Breastfeeding; n (\%) & 48/76 (63\%) & $11 / 20(55 \%)$ \\
\hline Contraceptive use; n (\%) & 38/97 (39\%) & $8 / 20(40 \%)$ \\
\hline Smoking; n (\%) & $17(17 \%)$ & $7(32 \%)$ \\
\hline \multicolumn{3}{|l|}{ Histology; n (\%) } \\
\hline Ductal & $80 / 90(89 \%)$ & 18/22 (82\%) \\
\hline Other & 10/90 (11\%) & $4 / 22(18 \%)$ \\
\hline \multicolumn{3}{|l|}{ Nuclear Grade; n (\%) } \\
\hline 2 & $31 / 80(39 \%)$ & 18/21 (86\%) \\
\hline 3 & $42 / 80(53 \%)$ & $2 / 21(10 \%)$ \\
\hline \multicolumn{3}{|l|}{ Stage; n (\%) } \\
\hline 0 & 1/101 (1\%) & 1. $(0)$ \\
\hline$|-| \mid$ & $57 / 101(56 \%)$ & $17 / 22(77)$ \\
\hline III & 39/101 (39\%) & $5 / 22(23)$ \\
\hline IV & 4/101 (4\%) & $0(0)$ \\
\hline \multicolumn{3}{|l|}{$\mathrm{T} ; \mathrm{n}(\%)$} \\
\hline $0-1$ & 29/100 (29\%) & $11 / 22(50)$ \\
\hline 2 & $37 / 100(37 \%)$ & $7 / 22(32)$ \\
\hline 3 & $25 / 100(25 \%)$ & $3 / 22(14)$ \\
\hline 4 & $9 / 100(9 \%)$ & $1 / 22(4)$ \\
\hline \multicolumn{3}{|l|}{ N; n (\%) } \\
\hline Positive & 59/99 (60\%) & $12 / 22(55 \%)$ \\
\hline \multicolumn{3}{|l|}{ IHC; n (\%) } \\
\hline $\mathrm{ER}(+)$ & $52 / 95(55 \%)$ & $22 / 22(100 \%)$ \\
\hline $\mathrm{PR}(+)$ & 47/95 (49\%) & $21 / 22(95 \%)$ \\
\hline $\operatorname{HER}(+)$ & 19/93 (20\%) & $5 / 22(23 \%)$ \\
\hline Triple negative & 30/93 (32\%) & $0(0 \%)$ \\
\hline \multicolumn{3}{|l|}{ Surgery type; n (\%) } \\
\hline Radical mastectomy & 80/99 (81\%) & $10 / 22(45 \%)$ \\
\hline Breast conservative & 18/99 (18\%) & $12 / 22(55 \%)$ \\
\hline
\end{tabular}

Table 1 Baseline characteristics of groups 1 \& 2; risk factors, tumor characteristics, and treatment (Continued)

\begin{tabular}{|c|c|c|}
\hline \multirow[t]{2}{*}{ Characteristic } & Group 1 & Group 2 \\
\hline & $n=101$ & $n=22$ \\
\hline \multicolumn{3}{|c|}{ Chemotherapy; n (\%) } \\
\hline Neoadjuvant & 28/100 (28\%) & 4/22 (18\%) \\
\hline Adjuvant & $62 / 100(62 \%)$ & $15 / 22(68 \%)$ \\
\hline Anthrac. based & 9/62 (15\%) & $7 / 15$ (40\%) \\
\hline Anthrac/Taxane. & 45/62 (72\%) & 6/15 (47\%) \\
\hline
\end{tabular}

$S D$ standard deviation, $I H C$ immunohistochemical analysis, ER estrogen receptor, $P R$ progesterone receptor; all means, and proportions were estimated for all the patients in each group, unless otherwise specified in the table

identified with NGS were re-sequenced by Sanger and all were true variants for a validation rate of $100 \%$. Two deletions, ex9-12del and ex16-17del accounted for 42.8\% among carriers in the familial-hereditary group, $21.4 \%$ (3/14) respectively. Two PV's (1 in BRCA1 and one in $B R C A 2)$ were detected in the positive control group. No PV's were detected in the 72 healthy women. Results of total variants are summarized in are reported in Additional file 1: Table S1.

A comparison of demographic and clinical characteristics between the mutation and non-mutation groups only revealed a difference in the frequency of breast feeding $(35.2 \%$ of mutated patients performed breastfeeding compared with $59.3 \%$ of non-mutated patients; $p=0.04$ ). Regarding tumor characteristics, the triple-negative subtype was more frequently observed in patients with BRCA PVs than in those without PVs $(65 \%$ vs. $22.6 \% ; p<0.001)$. The association of the triple-negative subtype with PVs of BRCA exhibited an OR of 6.4 (95\% CI, 2.22-18.70). Other clinical and tumor characteristics did not statistically differ between the mutation and non-mutation groups (Table 3).

\section{Discussion}

The university oncology center serves the northeast region of Mexico. At least 30\% come from other states and they are mostly low-income individuals who live in rural areas. So, the need for phone contact for participation and travel-related costs provoke low rates of participation, compared with the population found in the local database; however, the sample size was complete, as previously estimated.

Due to the lack of genetic characterization of BRCA genes in Mexico, 72 healthy women were included as control negative. Most of the previous studies are on Hispanics from diverse origins [7, 8]. There is scarce information in Mexico for healthy population. Local variants were not detected among healthy controls. Less information exists in Mexico about BRCA variants in this population. 


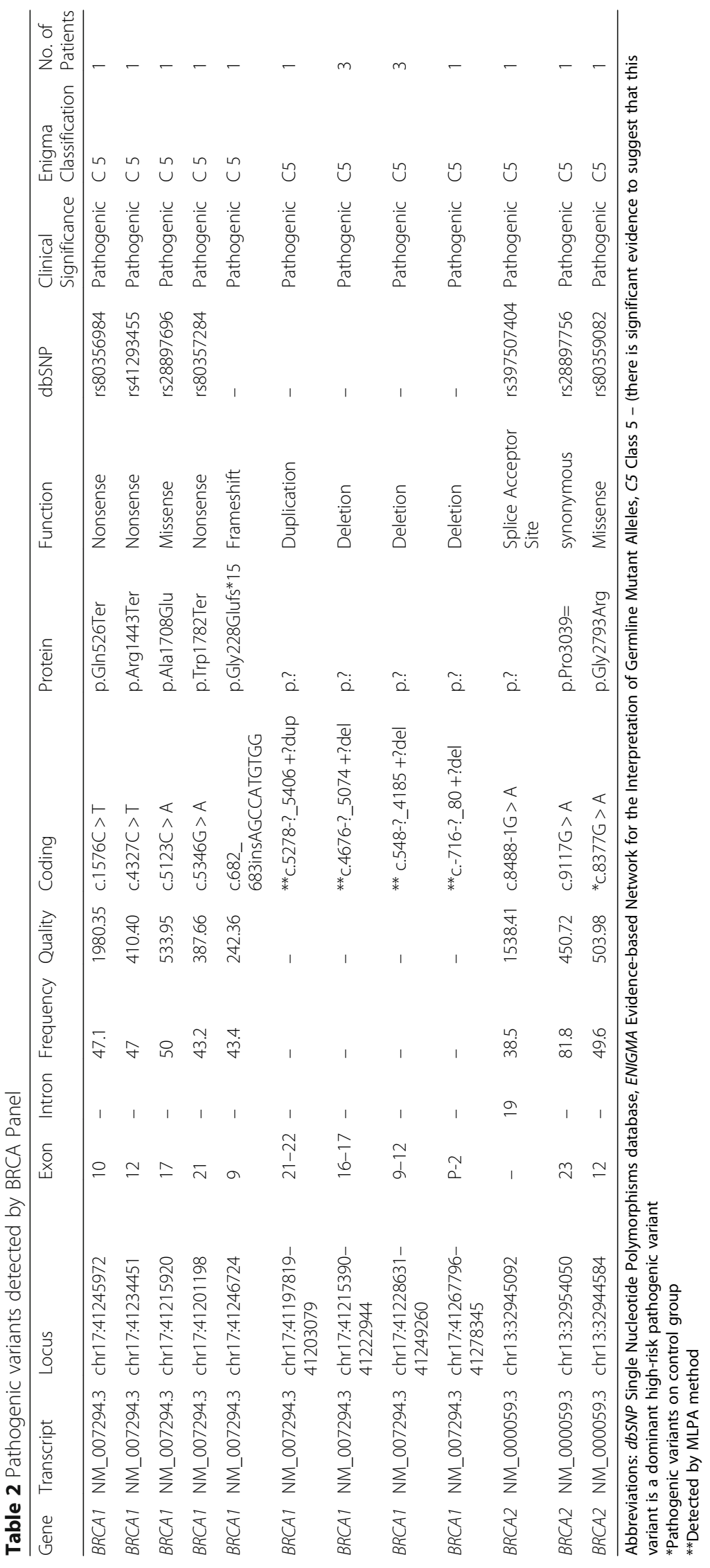


Table 3 Hereditary demographics, risk factors, and tumor characteristics

\begin{tabular}{|c|c|c|c|}
\hline Variable & $\begin{array}{l}\text { BRCA mutation } \\
\text { carriers } n=21\end{array}$ & $\begin{array}{l}\text { Non-carriers } \\
n=80\end{array}$ & \\
\hline Age: m (SD) & $35.6(4.5)$ & $37.3(5.4)$ & $p=0.18$ \\
\hline Family history of cancer & $66.6 \%$ & $50.0 \%$ & $p=0.17$ \\
\hline Body mass index, $m$ (SD) & $27.8(5.0)$ & $27.7(5.5)$ & $p=0.94$ \\
\hline Age at menarche $\mathrm{m}$ (SD) & $12.1(2.9)$ & $12.1(1.7)$ & $p=1.00$ \\
\hline No. pregnancy m (SD) & $2.4(2.1)$ & $2.3(1.5)$ & $p=0.80$ \\
\hline Nulligravid (\%) & $19.0 \%$ & $17.7 \%$ & $p=0.89$ \\
\hline Age at first birth, m (SD) & $20.7(4.9)$ & $21.6(5.6)$ & $p=0.50$ \\
\hline Breastfeeding \% & $35.2 \%$ & $59.3 \%$ & $p=0.04$ \\
\hline Contraception (\%) & $40 \%$ & $37.9 \%$ & $p=0.86$ \\
\hline Smoking (\%) & $14.2 \%$ & $17.5 \%$ & $p=0.72$ \\
\hline Histology, ductal. (\%) & $88 \%$ & $89 \%$ & $p=0.90$ \\
\hline \multicolumn{4}{|l|}{ Nuclear grade. (\%) } \\
\hline 2 & $27 \%$ & $41.5 \%$ & $p=0.28$ \\
\hline 3 & $67 \%$ & $49.2 \%$ & $p=0.22$ \\
\hline \multicolumn{4}{|l|}{ Stage. (\%) } \\
\hline 0 & $4.7 \%$ & $0 \%$ & $p=0.0002$ \\
\hline$|-| \mid$ & $71.4 \%$ & $52.5 \%$ & $p=0.12$ \\
\hline III & $23.8 \%$ & $42.5 \%$ & $p=0.11$ \\
\hline IV & $0 \%$ & $5 \%$ & $p=0.29$ \\
\hline \multicolumn{4}{|l|}{$\mathrm{T}$} \\
\hline $0-1$ & $19 \%$ & $31.6 \%$ & $p=0.26$ \\
\hline 2 & $57 \%$ & $31.6 \%$ & $p=0.03$ \\
\hline 3 & $19 \%$ & $26.6 \%$ & $p=0.47$ \\
\hline 4 & $4.7 \%$ & $10.2 \%$ & $p=0.43$ \\
\hline N Positive & $50 \%$ & $62 \%$ & $p=0.33$ \\
\hline \multicolumn{4}{|l|}{$\mathrm{IHC}$} \\
\hline ER $(+)$ & $30 \%$ & $60.5 \%$ & $p=0.01$ \\
\hline $\mathrm{PR}(+)$ & $30 \%$ & $53.9 \%$ & $p=0.058$ \\
\hline $\operatorname{HER}(+)$ & $16 \%$ & $21.6 \%$ & $p=0.57$ \\
\hline Triple Negative. & $65 \%$ & $22.6 \%$ & $p=<0.001$ \\
\hline
\end{tabular}

$m$ mean, $S D$ standard deviation, IHC immunohistochemical analysis, $E R$ estrogen receptor, $P R$ progesterone receptor

The frequency of PVs in BRCA1/2 genes reported by clinics that attend to high-genetic-risk populations in North America is approximately 9.3\% [15]; By contrast, the frequency of PVs reported in the Hispanic population from Southwestern United States is as high as 25\% [16]. In the present case-control study, a frequency of $13.8 \%$ of PVs was observed in a population from Northeastern Mexico, which is like that previously reported [17]. In Mexico, the frequency of PVs of these genes has been reported to be from 4 to $27 \%$, depending on the studied population (for example, cases with risk factors and sporadic cases) and tumor characteristics [17-20]. Particularly, among populations with familial/hereditary characteristics, the frequency was $10.2 \%$ in Mexico, which is not statistically different from our study $(p=0.14)$ [17].

Over 1,500 clinically significant PVs have been described for each BRCA gene [21, 22]. Among studies published in Mexican population 53 pathogenic genomic variants of BRCA1/2 (24 in patients with early onset or a family history of breast cancer, 28 in unselected populations, and one in both unselected populations) have been reported. Only one PV, a large genomic rearrangement (c.548-?.4185+?del), which is considered a founder mutation in Mexicans, was recurrent in different studies $[9,20]$. Torres-Mejía et al. and Villarreal et al. Reported the frequency of this PV was $1 \%$ or $22 \%$ among carriers and $9.4 \%$ or $42 \%$ among carriers, respectively. In our study we detected this PV in $2.9 \%$ or $21.4 \%$ among group 1 carriers\%. Inclusion criteria among these studies are different, going from an unselected population, triple negative in patients younger than $50 \mathrm{y}$ and in this study in an early breast cancer and/or family history. This data must be noticed because of the high spectrum of PVs in our population.

We discovered one PV, predicted to be deleterious, not previously reported; c.682_683insAGCCATGTGG; p.Gly228Glufs"15. This last PV was detected in an early age onset breast cancer patient, $33 \mathrm{y}$ at the time of diagnosis, with bilateral cancer and triple negative subtype. Two variants, p.Ser186Tyr and p.Thr1561Ile, are currently classified in several databases as benign. These two patients had early onset breast cancer at the age of $39 \mathrm{y}$ with HER overexpression and $37 \mathrm{y}$ with luminal subtype, none had family history. Nevertheless, according to the pathogenic predictors used in this study and considering the low frequency of these variants reported in 1000 Genomes Project, gnomAD, ExAc, we suggest further research for proper classification.

Some laboratories have been introducing multiplex assays, which analyze the most common genetic variants. In the present study, in addition to the founder genomic variant, all patients analyzed up to date were carriers of different PVs. From these data, we can infer that the use of these panels may provide missing information at least for Mexican populations.

New technologies such as NGS are currently being used for gene testing because they save time, are cost-effective, and have a higher sensitivity and specificity [23]. Nevertheless, it is important to use at least two different genomic technologies to rule out genomic variants, because as observed in this study, the use of MLPA enabled the identification of $38 \%$ of the PVs.

Because these technologies are not available in all clinical settings, clinical criteria should be considered to select patients for genetic testing. Recently, the criteria 
for hereditary breast cancer has been changing, with an expansion in the risk-related age range, family history, and pathologic characteristics [23] Patients with the triple-negative phenotype may even be of older age (> 50 years) [24]. In a previous study in Australia and Poland comprising patients unselected by age or family history of cancer, the prevalence was between 9.3 and $9.9 \%$ [25]. In a similar study of a Mexican population with a median age of 43 years (range, 23-50 years) and the triple-negative phenotype, the prevalence of PVs of BRCA was 23\% [26]. In this study, the frequency was as high as $43.3 \%$, representing $65 \%$ (OR, 6.4; 95\% CI, 2.2-18.7) of the patients with PVs, as mentioned previously. This finding indicates the importance of clinical aspects in decision making with regards to the need for gene testing.

Personal and environmental data are important characteristics for counseling and for decreasing the risk of breast cancer and other malignancies to some extent. Breastfeeding is considered an important protective factor for cancer development. In the present study, less proportion of women with PVs performed breastfeeding. Accordingly, it is important to recommend breastfeeding to carriers of BRCA PVs. This last modifiable risk factor has been described to be significant in decreasing the risk for breast cancer, with a relative risk of 0.63 (95\% CI, 0.460.86) in mutated BRCA1 populations [27]. To the best of our knowledge, this is the first study to compare the effect of breastfeeding on breast cancer of the young between carriers of $B R C A$ PVs and noncarriers in Mexico.

$B R C A$ gene status is important for the selection of treatment. The use of platinum analogs has shown more benefits in metastasis cases, with a favorable response of $54 \%$ compared with $19 \%$ for the use of other therapies [28]. Novel therapies that involve poly (ADP-ribose) polymerase inhibitors have shown advantages when used in combination with chemotherapy for BRCA-positive cases [29]. This highlights the need of gene testing not only for genetic counseling but also for treatment. In this study, therapy was not decided on the basis of the BRCA gene status.

\section{Conclusions}

In the present study, $B R C A$ PVs were detected with a frequency of $20 \%$ in a high-risk population, using Ion AmpliSeq BRCA1 and BRCA2 Panel together with MLPA. Because there is a high variability in the type and frequency of BRCA gene variants in the Mexican population, we propose the use of these technologies. We also state that clinical aspects can facilitate decision making regarding the need for $B R C A$ analysis. The triple-negative subtype has a good correlation with $B R C A$ mutations, so it is difficult to exclude this population from analysis. Strategies to promote a healthier environment must be included in the medical advice to patients.
Breastfeeding as a modifiable risk factor should be part of the analyses in future studies to determine the impact in high-risk groups of not only breast cancer, but also ovarian cancer.

\section{Additional file}

Additional file 1: Table S1. Genetic Database. Excel file with total data about genetic variants in BRCA1/2 about the three groups; Healthy, Sporadic, and Hereditary. (XLSX 83 kb)

\begin{abstract}
Abbreviations
ISPs: Ion Sphere Particles; MLPA: Multiple Ligation-dependent probe amplification; NGS: Next Generation Sequencing; ORs: Odds Ratios; PCR: Polymerase Chain Reaction; PV: Pathogenic Variant
\end{abstract}

\section{Acknowledgments}

The authors thank Antonio Diego de-la-Peña-Villarreal for the help in the recruitment process and database formulation of this study.

\section{Authors' contributions}

OAZV. Conception and design of study, analysis and interpretation of data, drafting of original manuscript, editing final manuscript, approval of the final version of the manuscript, data curation. LEMdV. Conception and design of study, interpretation of data, drafting of original manuscript, approval of the final version of the manuscript. LDCA. Analysis and interpretation of data, drafting of original manuscript, editing final manuscript, approval of the final version of the manuscript. JdJLT. Analysis and interpretation of data, drafting of original manuscript, editing final manuscript, approval of the final version of the manuscript, Data curation. IARS. Analysis and interpretation of data, drafting of original manuscript. JFGG. Conception of study, approval of the final version of the manuscript. MFNI. analysis and interpretation of data, drafting of original manuscript. DHB. Analysis and interpretation of data, editing final manuscript and approval of the final version of the manuscript. All authors have read and approved the manuscript.

\section{Funding}

There are no funding sources for this study.

Availability of data and materials

The datasets used by the authors are available on reasonable request to the corresponding author at laelmar@yahoo.com.mx.

\section{Ethics approval and consent to participate}

Institutional Review Board approval was obtained from The Bioethics Committee for Research in Health Science from the Hospital Universitario, Universidad Autónoma de Nuevo León, approved the study protocol, which was performed in accordance with the declaration of Helsinki and good clinical practices (Reference Number, GEN15-002). Written informed consent was obtained from each patient involved in this study.

Consent for publication

Not applicable.

\section{Competing interests}

The authors declare that we have no competing interests.

\section{Author details}

'Department of Genetics, Hospital Universitario "José E. González", Monterrey, Mexico. ${ }^{2}$ Department of Medical Oncology, Hospital Universitario "José E. González", Monterrey, Mexico. 
Received: 5 June 2018 Accepted: 17 July 2019

Published online: 22 July 2019

\section{References}

1. Ferlay J, Soerjomataram I, Dikshit R, et al. Cancer incidence and mortality worldwide: sources, methods, and major patterns in GLOBOCAN 2012 Int J Cancer. 2015;136:E359-86.

2. Couch FJ, Nathanson $\mathrm{KL}$, Offit $\mathrm{K}$. Two decades after BRCA: setting paradigms in personalized Cancer care and prevention. Science. 2014;343:1466-70. https://doi.org/10.1126/science.1251827.

3. Olopade OI, Grushko TA, Nanda R, Huo D. Advances in breast Cancer: pathways to personalized medicine. Clin Cancer Research. 2008;14:7988-99. https://doi.org/10.1158/1078-0432.

4. Hall JM, Lee MK, Newman B, et al. Linkage of early-onset familial breast cancer to chromosome 17q21. Science. 1990;250:1684-9.

5. Wooster R, Neuhausen SL, Mangion J, et al. Localization of a breast cancer susceptibility gene, BRCA2, to chromosome 13q12-13. Science. 1994:265:2088-90

6. Mavaddat N, Peock S, Frost $\mathrm{D}$, et al. Cancer risks for BRCA1and BRCA2 mutation carriers: results from prospective analysis of eMBrAce. J Natl Cancer Inst. 2013;105:812-22.

7. Ossa CA, Torres D. Founder and recurrent mutations in BRCA1 and BRCA2 genes in Latin American countries: state of the art and literature review. Oncologist. 2016;21:1-8.

8. Weitzel JN, Clague J, Martir-Negron A, et al. Prevalence and type of BRCA mutations in Hispanics undergoing genetic cancer risk assessment in the southwestern United States: a report from the clinical cancer genetics community research network. J Clin Oncol. 2012;31:210-6.

9. Villarreal-Garza C, Alvarez-Gomez RM, Plascencia CP, et al. Significant clinical impact of recurrent BRCA1 and BRCA2 mutations in Mexico. Cancer. 2015;121:372-8. https://doi.org/10.1002/cncr.29058.

10. Partridge A, Pagani $\mathrm{O}$, Abulkhair $\mathrm{O}$, Aebi S, Amant F. First international consensus guidelines for breast cancer in young women (BCY). Breast. 2014;23:209-20.

11. Sin HP, Kreipe $\mathrm{H}$. A brief overview of the $\mathrm{WHO}$ classification of breast tumors, $4^{\text {th }}$ edition, focusing on issues and updates from the $3^{\text {rd }}$ edition. Breast Care. 2013:8:149-54.

12. Hammond ME, Hayes DF, Dowsett M, et al. American Society of Clinical Oncology / College of American Pathologists guideline recommendations for immune-histochemical testing of estrogen and progesterone receptors in breast cancer. J Clin Oncol. 2010;28:2784-95.

13. Wolff AC, Hammond ME, Hicks DG, et al. Recommendations for human epidermal growth factor receptor 2 testing in breast cancer: American Society of Clinical Oncology/College of American Pathologists clinical practice guideline update. J Clin Oncol. 2013;31:3997-4013. https://doi.org/1 $0.1200 / J C O .2013 .50 .9984$

14. Balmana J, Diez O, Rubio IT, Cardoso F. BRCA in breast cancer: ESMO clinical practice guidelines. Ann Oncol. 2011;22:vi31-4.

15. Tung N, Batelli C, Allen B. Frequency of mutations in individuals with breast Cancer referred for BRCA1 and BRCA2 testing using next generation sequencing with a 25-gene panel. Cancer. 2015;121:25-33.

16. Weitzel JN, Clague J, Martir-Negron A. Prevalence and type of BRCA mutations in Hispanics undergoing genetic Cancer risk assessment in the southwestern United States: a report from the clinical Cancer genetics community research network. J Clin Oncol. 2012;31:210-6.

17. Vaca-Paniagua F, Alvarez-Gomez R, Fragoso-Ontiveros V. Full-exon pyrosequencing screening of BRCA germline mutations in Mexican women with inherited breast and ovarian Cancer. PLoS One. 2012;7:e37432.

18. Ruiz-Flores P, Sinilnikova O, Badzioch M (2002) BRCA1 and BRCA2 mutation analysis of early onset and familial breast Cancer cases in Mexico. Hum Mutat 20:474-475. [PubMed: 12442275].

19. Calderón-Garcidueñas A, Ruiz-Flores P, Cerda-Flores R. Clinical follow up of Mexican women with early onset of breast cancer and mutations in the BRCA1 and BRCA2 genes. Salud Publica Mex. 2005;47:110-5.

20. Torres-Mejia G, Royer R, Llacuachaqui M, Akbari M, Giuliano A. Recurrent BRCA1 and BRCA2 mutations in Mexican women with breast Cancer. Cancer Epidemiol Biomark Prev. 2014:24:498-505.

21. National Center for Biothechnology Information (2017) Variation Viewer. Version 1.5.5. https://www.ncbi.nlm.nih.gov/variationviewer/view/?q=672\%5 Bgeneid\%5D\&assm=GCF_000001405.25. Accessed 22 May 2017
22. National Center for Biotechnology Information (2017) Variation Viewer Version 1.5.5. http://www.ncbi.nlm.nih.gov/variation/view/?q=675\%5 Bgeneid\%5D\&assm=GCF_000001405.25. Accessed 22 May 2017.

23. The NCCN Clinical Practice Guidelines in Oncology ${ }^{\top \mathrm{M}}$ : Genetic/Familial HighRisk Assessment: Breast and Ovarian. Version 2.2017 - December 7, 2016. http://www.nccn.org/. Accessed 2 Feb 2017.

24. Rhiem K, Engel C, Engel J, Niederacher D, Sutter C (2016) BRCA1/2 mutation prevalence in triple negative breast cancer patients without family history of breast and ovarian cancer. J Clin Oncol 34:1090-1090.

25. Wong-Brown MW, Meldrum CJ, Carpenter JE. Prevalence of BRCA1 and BRCA2 germline mutations in patients with triple negative breast cancer. Breast Cancer Res Treat. 2015;150:71-80. https://doi.org/10.1007/s10549-015-3293-7.

26. Villarreal-Garza C, Weitzel J, Llacuachaqui M, Sifuentes E, Magallanes-Hoyos $M$. The prevalence of BRCA1 and BRCA2 mutations among young Mexican women with triple-negative breast cancer. Breast Cancer Res Treat. 2015;150:389-94.

27. Friebel T, Domchek S, Rebbeck T. Modifiers of Cancer risk in BRCA1 and BRCA2 mutation carriers: systematic review and meta-analysis. JNCl, Journal of the National Cancer Institute. 2014;106(6):dju091.

28. Isakoff SJ, Mayer EL, He L. TBCRC009: A Multicenter Phase II Clinical Trial of Platinum Monotherapy with Biomarker Assessment in Metastatic TripleNegative Breast Cancer. J Clin Oncol. 2015:33:1902-9. https://doi.org/10.12 00/JCO.2014.57.6660

29. O'Shaughnessy J, Shwartzberg L, Danso MA. Phase III study of iniparib plus gemcitabine and carboplatin versus gemcitabine and carboplatin in patients with metastatic triple-negative breast cancer. J Clin Oncol. 2014;32:3840-7. https://doi.org/10.1200/JCO.2014.55.2984.

\section{Publisher's Note}

Springer Nature remains neutral with regard to jurisdictional claims in published maps and institutional affiliations.

Ready to submit your research? Choose BMC and benefit from:

- fast, convenient online submission

- thorough peer review by experienced researchers in your field

- rapid publication on acceptance

- support for research data, including large and complex data types

- gold Open Access which fosters wider collaboration and increased citations

- maximum visibility for your research: over $100 \mathrm{M}$ website views per year

At BMC, research is always in progress.

Learn more biomedcentral.com/submissions 